\title{
PROSPECTIVE STUDY OF ADENOVIRUS ANTIGEN DETECTION IN EYE SWABS BY RADIOIMMUNE DOT-BLOT
}

\author{
D. J. MORRIS ${ }^{1}$, P. E. KLAPPER ${ }^{1}$, R. KILLOUGH ${ }^{1}$, A. S. BAILEY ${ }^{1}$, J. NELSON ${ }^{1}$ and \\ A. B. TULLO ${ }^{2}$ \\ Manchester
}

\begin{abstract}
SUMMARY
Rapid laboratory diagnosis of ocular adenovirus infection is crucial in the containment of nosocomial transmission of the virus. In a large prospective study of adenovirus assay in eye swabs, antigen detection by radioimmune dot-blot (turnaround time 72 hours) achieved a sensitivity of $67 \%(239 / 355)$ and a specificity of $93 \%(3065 / 3285)$ in comparison with virus culture (median turnaround time 14 days). When specimens weakly reactive for adenovirus antigen, or equally reactive for both adenovirus antigen and Chlamydia trachomatis antigen, were considered falsely reactive in the adenovirus test, the sensitivity of the latter was reduced and false positive reactions were only marginally less frequent. The radioimmune dot-blot provides a more rapid diagnosis of ocular adenovirus infection than virus culture, but the high risk of false negative and in particular false positive results limits its clinical utility.
\end{abstract}

Adenovirus eye disease manifests as epidemic or sporadic keratoconjunctivitis, follicular conjunctivitis or pharyngoconjunctival fever. ${ }^{1}$ Epidemic disease, associated with serotypes 8,19 and 37 of subgenus $D$, can occur in large outbreaks in eye hospitals. ${ }^{1,2}$ Sporadic cases are caused by a variety of adenovirus serotypes, including 3, 7, 14 (subgenus B), 1, 2, 5, 6 (subgenus C), 9, 10 (subgenus D), or 4 (subgenus E). ${ }^{1}$

Laboratory diagnosis of ocular adenovirus infection relies on antibody assay in serum, virus isolation in cell culture from eye swabs, or viral antigen detection in similar specimens by enzyme-linked immunosorbent assay. ${ }^{1,3,4}$ The first two methods are retrospective, virus isolation taking a mean of

From: ${ }^{1}$ Clinical Virology Laboratory, Manchester Royal Infirmary; ${ }^{2}$ Manchester Royal Eye Hospital, Manchester, UK.

Correspondence to: Dr D. J. Morris, Clinical Virology Laboratory, 3rd Floor, Clinical Services Building, Manchester Royal Infirmary, Oxford Road, Manchester M13 9WL, UK.
14 days. Moreover, given the large numbers of adenovirus serotypes, neutralising or haemagglutinating antibody tests can only be used if the likely infecting serotype is known. ${ }^{1}$ We recently described an adenovirus antigen detection assay which provided a rapid result (within 72 hours), and achieved a sensitivity of $85 \%$ in a preliminary study. ${ }^{5}$ We now report a large prospective evaluation of this radioimmune dot-blot test (IDBT) on 3760 conjunctival swabs.

\section{MATERIALS AND METHODS}

All conjunctival swabs received between January 1990 and September 1993 were included if they had been tested for adenovirus by both IDBT and virus isolation. Cotton-tipped swabs were placed in $3 \mathrm{ml}$ transport medium (Hanks' balanced salt solution with $10 \%[\mathrm{v} / \mathrm{v}$ ] fetal calf serum, $2.5 \%$ [w/v] sodium bicarbonate, 200 international units $/ \mathrm{ml}$ penicillin $\mathrm{G}$, $200 \mathrm{mg} / \mathrm{ml}$ streptomycin and $5 \mathrm{mg} / \mathrm{ml}$ amphotericin B), and transported to the laboratory at ambient temperature.

Virus isolation on monolayer cultures of Hep-2, Vero and human embryo lung fibroblasts, and adenovirus typing by neutralisation, were done as previously described. ${ }^{5}$

The adenovirus IDBT was performed approximately twice weekly on residual samples stored at $4{ }^{\circ} \mathrm{C} .{ }^{5}$ Aliquots $(0.2 \mathrm{ml})$ were heat-inactivated $\left(56^{\circ} \mathrm{C}\right.$ for 30 minutes) and then dot-blotted in duplicate onto a pre-moistened nitrocellulose membrane. After blocking with $5 \%$ skimmed milk in phosphate-buffered saline (PBSM), the membrane was probed with PBSM containing 50-70 $\times 10^{3}$ counts/ $\mathrm{min} / \mathrm{ml}{ }^{125}$ I-labelled mouse monoclonal antibody specific for a genus reactive epitope on the adenovirus hexon protein. ${ }^{6}$ Binding of the monoclonal antibody was revealed following autoradiography ( 48 hours at $-70^{\circ} \mathrm{C}$ ). To check the specificity 
Table I. Adenovirus positivity rates in cell culture for eye swabs showing various combinations of reactivity in the adenovirus and chlamydial IDBT

\begin{tabular}{ccc}
\hline $\begin{array}{c}\text { Adenovirus } \\
\text { IDBT }^{\mathrm{a}}\end{array}$ & $\begin{array}{c}\text { Chlamydial } \\
\text { IDBT }^{\mathrm{a}}\end{array}$ & $\begin{array}{c}\text { Proportion of adenovirus } \\
\text { positive in virus isolation }\end{array}$ \\
\hline+ & - & $90 / 135(67 \%)$ \\
+ & \pm & $38 / 62(61 \%)$ \\
++ & + & $39 / 50(78 \%)$ \\
\pm & - & $42 / 94(45 \%)$ \\
\pm & \pm & $19 / 81(23 \%)$ \\
+ & + & $10 / 24(42 \%)$ \\
\pm & + & $1 / 13(8 \%)$ \\
\hline
\end{tabular}

${ }^{a}$ Results on autoradiograph were scored as: - , negative; \pm , weak positive (incomplete circle or circular darkening less intense than control for the same antigen); + , positive (dark circle at least as intense as the control for the same antigen); ++ , strong positive (dark circle more intense than the control for the same antigen and more intense than the circle for the other antigen).

of any reaction in the adenovirus IDBT, the conjunctival swab transport medium was also tested for Chlamydia trachomatis lipopolysaccharide antigen using an IDBT identical in all respects to the adenovirus assay except for reliance on a Chlamydiaspecific mouse monoclonal antibody. ${ }^{7}$ Complete darkened circles on the autoradiograph were considered to indicate reactivity with the relevant antibody. In each assay doubling dilutions of a positive control were included: either adenovirus type 2 infected Hep-2 cells or Chlamydia trachomatis infected McCoy cells. A sample was considered reactive if the circle it produced was greater in intensity than the circle which corresponded to 25 tissue culture infectious dose -50 of adenovirus type $2^{5}$ or 100 inclusion-forming units of Chlamydia trachomatis. ${ }^{8}$ Incomplete circles or circles of lower intensity than these controls were interpreted as weakly positive reactions. If both reactions were positive, the more intense was classified as strongly positive.

Statistical analysis relied on chi-squared or Fisher's exact tests, as appropriate, $p$ values being two-tailed.

\section{RESULTS}

Eye swabs yielding herpes simplex virus $(n=119)$ or varicella zoster virus $(n=1)$ in cell culture were excluded from further analyses. Of the 239 adenovirus IDBT and culture positive specimens, $62(26 \%)$ reacted only weakly for adenovirus antigen, and 107 $(45 \%)$ reacted simultaneously for adenovirus and Chlamydia trachomatis. Compared with virus isolation, the adenovirus IDBT achieved a sensitivity of $67 \%(239 / 355)$, a specificity of $93 \%(3065 / 3285)$, a positive predictive value of $52 \%(239 / 459)$ and a negative predictive value of $96 \%$ (3065/3181) if all reactivity for adenovirus antigen of this IDBT was considered to reflect the presence of that antigen. If the specimens producing weakly reactive results in the adenovirus IDBT were considered negative for adenovirus antigen the sensitivity of the rapid assay was lower than before $(177 / 355,50 \% ; p<0.001)$, the positive predictive value $(177 / 271,65 \%)$ increased $(p<0.001)$, but the specificity $(3127 / 3285.95 \%)$ and the negative predictive value $(3127 / 3305,95 \%)$ were virtually unchanged. If a specimen was considered positive for adenovirus antigen only when it reacted more intensely with anti-adenovirus antibody than with the chlamydial antibody, the sensitivity was again reduced $(209 / 355,59 \% ; p=0.008)$, the positive predictive value $(209 / 341,61 \%)$ increased $(p<0.01)$, and there was little effect on the specificity (3095/ $3285,94 \%)$ and the negative predictive value (3095/ $3241,95 \%)$.

Specimens more strongly reactive in the adenovirus than the chlamydial antigen test were more likely to be positive for adenovirus in cell culture than were specimens equally reactive in the two tests or more strongly reactive for chlamydial antigen $(209 / 341[62 \%]$ versus 30/118 [25\%], $p<0.001$ ) (Table I). When all reactivity with anti-adenovirus antibody was considered positive and the Chlamydia result was not taken into account, the sensitivity of the adenovirus IDBT was lower with subgenus $\mathrm{C}$ adenovirus strains than with other typed adenoviruses (Table II) $(p<0.001)$, with specimens positive for serotype $7(p=0.03)$ or 8 by virus isolation $(p=0.009)$, and possibly with specimens similarly positive for adenoviruses of subgenera B $(p=0.06)$ or $\mathrm{E}(p=0.07)$. Low sensitivity was seen with all four adenovirus subgenus $\mathrm{C}$ serotypes (Table II).

\section{DISCUSSION}

Overall the performance of the adenovirus IDBT was disappointing, with a sensitivity of only $67 \%$ in comparison with the $85 \%$ reported previously from our laboratory. ${ }^{5}$ Other workers reported a sensitivity for antigen detection by enzyme-linked immunosorbent assay of only $75 \%$ in comparison with virus isolation.,4 Differences between our initial and present studies included a change in the temperature at which specimens were stored prior to antigen testing $\left(-40^{\circ} \mathrm{C}\right.$ versus $\left.4{ }^{\circ} \mathrm{C}\right)$, and doubling of the

Table II. Adenovirus subgenera and serotypes and sensitivity of adenovirus IDBT in comparison with virus isolation

\begin{tabular}{|c|c|c|c|}
\hline \multirow{3}{*}{$\frac{\text { Subgenus }}{\mathrm{B}}$} & \multirow{3}{*}{$\begin{array}{c}\text { Serotype } \\
3,7,14\end{array}$} & \multicolumn{2}{|c|}{ Sensitivity } \\
\hline & & \multicolumn{2}{|c|}{$92 / 134(69 \%)$} \\
\hline & & $62 / 95$ & $(65 \%)$ \\
\hline & 7 & $28 / 36$ & $(78 \%)$ \\
\hline & 14 & $2 / 3$ & $(67 \%)$ \\
\hline \multirow[t]{5}{*}{$\mathrm{C}$} & $1,2,5,6$ & $13 / 26$ & $(50 \%)$ \\
\hline & 1 & $5 / 11$ & $(45 \%)$ \\
\hline & 2 & $5 / 9$ & $(56 \%)$ \\
\hline & 5 & $1 / 2$ & $(50 \%)$ \\
\hline & 6 & $2 / 4$ & $(50 \%)$ \\
\hline \multirow[t]{4}{*}{ D } & $8,9,10$ & $62 / 95$ & $(65 \%)$ \\
\hline & 8 & $24 / 28$ & $(86 \%)$ \\
\hline & 9 & $0 / 1$ & $(0 \%)$ \\
\hline & 10 & $38 / 66$ & (58\%) \\
\hline E & 4 & $47 / 66$ & $(71 \%)$ \\
\hline Untyped & & $25 / 34$ & $(74 \%)$ \\
\hline
\end{tabular}

Chlamydia IDBT results were disregarded in this analysis. 
transport medium volume from 1.5 to $3.0 \mathrm{ml}$ to guarantee an adequate volume for all tests. We did not determine whether either of these alterations reduced the assay sensitivity. The sensitivity in the current study was probably a more accurate reflection of the test performance than the earlier results, given the difference in specimen numbers (3760 versus 754). Only in the larger study was a subgenusspecific difference in IDBT performance identified, with low sensitivity for subgenus $C$ strains but excellent sensitivity for type 8 strains. This difference could have reflected the presence of differing amounts of viral antigen in the conjunctival secretions of patients infected with adenoviruses of different subgenera. The relatively high specificity reported earlier $(92 \%)^{5}$ was maintained $(93 \%)$, but the low positive predictive value compromised the overall clinical usefulness of the test even when weak reactivity for adenovirus antigen was considered false. Nevertheless, the adenovirus IDBT proved valuable in containing a recent outbreak of adenovirus type 8 keratoconjunctivitis.

Immune dot-blots where the total protein in a sample is immobilised on a nitrocellulose membrane may give false positive results when specimens contain staphylococcal protein A. ${ }^{7}$ Specimen pretreatment with proteinase $\mathrm{K}$ circumvented this problem in an IDBT for Chlamydia trachomatis lipopolysaccharide antigen, ${ }^{8}$ but could not be applied in the adenovirus assay since a protein antigen was assayed. In a previous report we recommended simultaneous testing of eye swabs for adenovirus and either chlamydial or herpes simplex virus antigens to differentiate true positive reactivity (positive result only for adenovirus) from false reactivity (positive results for both antigens). ${ }^{5}$ In the present study such an approach led to a reduction in the sensitivity of the adenovirus IDBT, and increased its positive predictive value to only $61 \%$. Specimens selectively reactive for adenovirus antigen were more likely to yield adenovirus in cell culture than those equally reactive for both adenovirus and chlamydial antigens, but $8-42 \%$ of specimens in the latter category (depending on the individual antigen results; Table I) yielded adenovirus in virus isolation.

To determine the significance of discrepant results in the adenovirus and chlamydial antigen detection and adenovirus culture tests, adenovirus DNA was sought in a small number of specimens using a genusreactive polymerase chain reaction (PCR). ${ }^{10}$ Adenovirus DNA was detected by PCR in 0 of 6 specimens reactive in both IDBTs, and in 3 of 7 specimens reactive only in the adenovirus antigen assay when virus isolation gave a negative result (A. S. Bailey, unpublished observations). The PCR result therefore suggested that even a positive reaction in the adenovirus IDBT and a negative result in the
Chlamydia assay did not always indicate the presence of adenovirus in the clinical specimen. However, the specimens equally reactive in both IDBTs or more strongly reactive in the chlamydial test were not all falsely positive in the adenovirus antigen assay, as adenoviruses were detected in cell culture from 30 of 118 such specimens.

In the late 1980s we developed two assays intended inter alia to improve the diagnosis of ocular infection. Assay of Chlamydia trachomatis lipopolysaccharide antigen proved more sensitive than chlamydial culture, and antigen-positive culture-negative specimens mostly contained chlamydial DNA detectable using the PCR. ${ }^{11}$ In contrast the adenovirus antigen detection assay was less sensitive than cell culture and generated both uninterpretable low positive and apparently false positive results. This difference could in part have reflected differences in the amount of antigen in eye swabs during the two infections. More importantly, however, the restriction of proteinase $\mathrm{K}$ specimen pretreatment to the chlamydial assay was crucial because this increased both sensitivity and specificity. ${ }^{8}$ Use of $\mathrm{Fab}_{2}$ fragments of the adenovirus monoclonal antibody for radiolabelling might have increased the specificity of adenovirus assay because protein $\mathrm{A}$ binds to the $\mathrm{Fc}$ portion of immunoglobulin. ${ }^{12}$ However, our unpublished observations indicated that digestion of this antibody with pepsin resulted in poor yield of antibody, and that the resultant $\mathrm{Fab}_{2}$ fragment was inactivated on radioiodination. Presumably the tyrosine residue enabling radioiodination of the adenovirus IgG monoclonal antibody with retention of biological activity resides on the Fc portion of antibody molecule. Attempted circumvention of this problem through the use of non-isotopic labelling (biotin-avidin labelling with dye precipitation: 5-bromo-4-chloro-2-indolyl phosphate dye) did not produce an assay of adequate sensitivity and specificity.

Chlamydia antigen detection using the IDBT has an established place in the diagnosis of ocular infection, ${ }^{11}$ but the role of adenovirus antigen detection by IDBT is less certain. We and others have recently reported that DNA amplification using the PCR is more sensitive than antigen assays for the detection of adenoviruses in eye swabs. ${ }^{13,14}$ The former technique may generate false positive results due to specimen contamination with exogenous adenovirus DNA or reaction product. ${ }^{13}$ The present study nonetheless indicates that the IDBT has such a low sensitivity and such a high false positivity rate that its replacement by the PCR for the rapid diagnosis of ocular adenovirus infection is inevitable.

We are grateful to Ian Sharp, Central Public Health Laboratory, Colindale, London, for supplying the adenovirus monoclonal antibody.

Key words: Adenovirus, Antigen detection, Conjunctivitis, Dotblot test, Rapid diagnosis. 


\section{REFERENCES}

1. Wadell G. Adenoviruses. In: Zuckerman AJ, Banatvala JE, Pattison JR, editors. Principles and practice of clinical virology. Chichester: Wiley, 1987:251-74.

2. Keenlyside RA, Hierholzer JC, D'Angelo LJ. Keratoconjunctivitis associated with adenovirus type 37: an extended outbreak in an ophthalmologist's office. J Infect Dis 1983;147:191-8.

3. Wiley L, Springer D, Kowalski RP, Arffa R, Roat MI, Thoft RA, Gordon VJ. Rapid diagnostic tests for ocular adenovirus. Ophthalmology 1988;95:431-3.

4. Kowalski RP, Gordon VJ. Comparison of direct rapid tests for the detection of adenovirus antigen in routine conjunctival specimens. Ophthalmology 1989;96:1106-8.

5. Killough R, Klapper PE, Bailey AS, Sharp IR, Tullo A, Richmond SJ. An immune dot-blot technique for the diagnosis of ocular adenovirus infection. J Virol Methods 1990;30:197-204.

6. Sharp IR, Bailey AS. Development of a diagnostic reagent for the detection of adenovirus antigen by immunofluorescence. Analyt Proc R Soc Chem 1988; 25:129-31.

7. Storey CC, Mearns G, Richmond SJ. Immune dot-blot technique for diagnosing infection with Chlamydia trachomatis. Genitourin Med 1987;63:375-9.

8. Mearns G, Richmond SJ, Storey CC. Sensitive immune dot-blot test for diagnosis of Chlamydia trachomatis infection. J Clin Microbiol 1988;26:1810-3.
9. Ankers HE, Klapper PE, Cleator GM, Bailey AS, Tullo AB. The role of a rapid diagnostic test (adenovirus immune dot-blot) in the control of an outbreak of adenovirus type 8 keratoconjunctivitis. Eye 1993;7 Suppl:15-7.

10. Turner PC, Bailey AS, Cooper RJ, Morris DJ. The polymerase chain reaction for detecting adenovirus DNA in formalin-fixed paraffin-embedded tissue obtained post-mortem. J Infect 1993;27:43-6.

11. Bishop PN, Tullo AB, Killough R, Richmond SJ. An immune dot-blot test for the diagnosis of ocular infection with Chlamydia trachomatis. Eye 1991;5:305-8.

12. Cremer NE, Riggs RL. Immunoglobulin classes and viral diagnosis. In: Lennette EH, Schmidt NJ, editors. Diagnostic procedures for viral, rickettsial and chlamydial infections. Wáshington, DC: American Public Health Association, 1979:191-208.

13. Morris DJ, Bailey AS, Cooper RJ, Turner PC, Jackson R, Corbitt G, Tullo AB. Polymerase chain reaction for detection of ocular adenovirus infection. J Med Virol, in press.

14. Kinchington PR, Turse SE, Kowalski RP, Gordon VJ. Use of polymerase chain amplification reaction for the detection of adenoviruses in ocular swab specimens. Invest Ophthalmol Vis Sci 1994;35:4126-34. 